\title{
Brr2 is a splicing fidelity factor
}

\author{
Megan Mayerle ${ }^{1,2} \&$ Christine Guthrie ${ }^{1,3}$ \\ 1 Department of Biochemistry and Biophysics, University of California, San Francisco, San Francisco, CA, USA \\ 2 MeganMayerle82@gmail.com \\ 3 ChristineGuthrie@gmail.com
}

\begin{abstract}
Many spliceosomal DExD/H box helicases act as fidelity factors during pre-mRNA splicing, promoting on-pathway interactions while simultaneously minimizing errors. Mutations linked to Retinitis Pigmentosa (RP), a form of heritable blindness, map to key domains of spliceosomal helicase Brr2 (SNRNP200 in humans). Previous data show that such mutations negatively impact spliceosome activation, likely due to defects in brr2-RP RNA binding, helicase, and ATPase activities. Furthermore, data from human reporter constructs suggest that brr2-RP might impact $5^{\prime}$ splice site selection. Here we undertake a systematic analysis of $b r r 2-R P$ effects on splicing fidelity. We show that a subset of $b r r 2-R P$ mutants exhibit intron retention in vivo. Furthermore, brr2-RP mutants display hyperaccurate and/or error-prone splicing of a variety of splicing reporters. Branch-site fidelity is particularly impacted in this reporter assay. In addition, multiple brr2-RP alleles genetically interact with prp16 alleles known to impact the fidelity of branch site selection. Together these data implicate Brr2 in the fidelity of branch-site selection, and suggest that RP results not just from defects in spliceosome activation, but also from fidelity defects arising throughout the splicing cycle and in splicing fidelity.
\end{abstract}

\section{Introduction}

Splicing is an essential step in gene expression wherein the spliceosome identifies and catalyzes the removal of non-coding introns and the joining of flanking exons from pre-mRNAs (Will and Lührmann 2011). Splicing is an inherently high-fidelity reaction, with an error rate as low as 1 in every 100,000 splicing events (Fox-Walsh and Hertel 2009). This high level of fidelity results from several independent quality-control pathways as well as from fidelity mechanisms enacted by the spliceosome's own RNA and protein components, particularly the $\mathrm{DExD} / \mathrm{H}$ box ATPases. Spliceosomal DExD/H box ATPases, such as Prp5, Prp16, and Prp22, employ kinetic proofreading to both antagonize the splicing of suboptimal, low fidelity substrates and to promote the splicing of optimal, high fidelity substrates (Semlow and Staley 2012).

Spliceosomal protein Brr2 is a DEIH box ATPase and the most highly processive spliceosomal helicase(Raghunathan and Guthrie 1998). Initial Brr2 characterizations focused on its role in unwinding the snRNA duplexes during spliceosome activation (Lauber et al. 1996; Raghunathan and Guthrie 1998), however Brr2 has since been implicated in multiple steps of the splicing cycle: during the transition between the $1^{\text {st }}$ and $2^{\text {nd }}$ catalytic steps (Hahn et al. 2012; Mayerle and Guthrie 2016), during spliceosome disassembly (Small et al. 2006), and in pre-mRNA discard from the spliceosome (Chen et al. 2013). Brr2's helicase and ATPase activities are highly regulated, via both intramolecular and extramolecular mechanisms, to ensure that Brr2 acts only at appropriate points in the splicing cycle(Absmeier et al. 2017).

Brr2 is one of multiple spliceosomal proteins implicated in Retinitis Pigmentosa (RP), a hereditary form of blindness(Růžičková and Staněk 2017). Point mutations identified as causal in RP tend to map to highly conserved, and thus likely functionally important, amino acids. Numerous groups have created yeast strains wherein the genes coding for splicing factors have homologous mutations to those 
implicated in RP, and used such yeast to probe spliceosome assembly and function (reviewed in (Růžičková and Staněk 2017; Daiger et al. 2014).

Here we show that $b r r 2-R P$ yeast impact a wide variety of Brr2 functions in the cell. Most importantly, we show that brr2-RP affect splicing efficiency and fidelity, exhibiting both errorprone and hyperaccurate splicing depending upon the pre-mRNA substrate. We also genetically link Brr2 and Prp16 ATPase activities. While the underlying mechanism remains unclear, together our data clearly implicate Brr2 in splicing fidelity, and suggest that splicing fidelity, not just splicing efficiency, may be impacted in RP.

\section{Results and Discussion}

\section{Alleles of BRR2 linked to retinitis pigmentosa impact multiple Brr2 functions both in vitro and in vivo}

We created $S$. cerevesiae strains bearing alleles of $B R R 2$ that are homologous to specific alleles of SNRNP200 implicated in RP pathogenesis in humans(Růžičková and Staněk 2017). Working in yeast allows us to take advantage of the extensive array of genetic, biochemical, and molecular techniques available within this system to provide mechanistic insights into how brr2-RP impact Brr2 function. brr2-RP alleles brr2-C520R and brr2-Q904E both map to Brr2's ATPase domain, while alleles N1104A, R1107A, and $R 1107 \mathrm{~L}$ map to the ratchet helix in Brr2's helicase domain, both of which are essential for Brr2 function (Figure 1A)(Santos et al. 2012; Nguyen et al. 2013). The brr2-R1107M allele included in our studies has not been associated with RP; it was a cloning error that serves as a control. In all of these strains, the genomic copy of $B R R 2$ has been deleted and Brr2 and Brr2-RP variants are expressed, under the control of their endogenous promoter, from a pRS313 plasmid.

Given that Brr2 is an essential protein(Noble and Guthrie 1996; Raghunathan and Guthrie 1998), we initially set out to assess how brr2-RP impact Figure 1

A

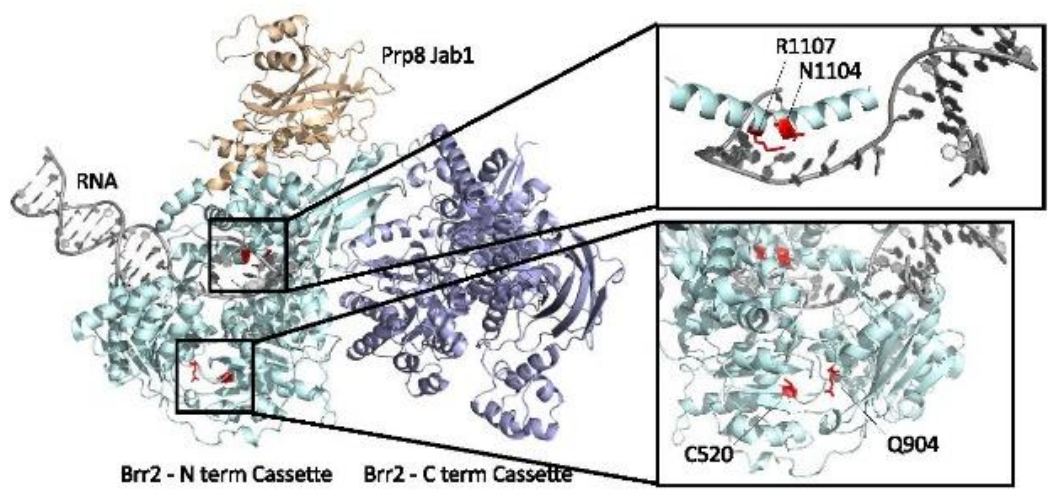

B

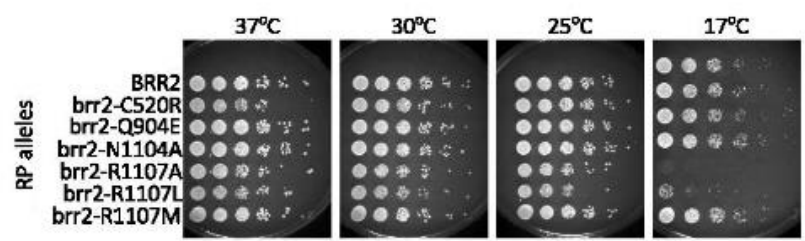

\begin{tabular}{|c|c|c|c|c|c|c|}
\hline $\begin{array}{c}\text { RP allele } \\
\text { (human/yeast) }\end{array}$ & $\begin{array}{c}\text { expression } \\
\text { level }\end{array}$ & growth & $\begin{array}{l}\text { helicase } \\
\text { activity }\end{array}$ & $\begin{array}{l}\text { ATPase } \\
\text { activity }\end{array}$ & $\begin{array}{l}\text { U4/U6 } \\
\text { affinity }\end{array}$ & $\begin{array}{c}\text { snRNp } \\
\text { level }\end{array}$ \\
\hline C502/502R & - & ts & - & - & - & - \\
\hline Q885/904E & - & WT & defect & defect & - & - \\
\hline S1087/N1104A & - & WT & WT & - & - & WT \\
\hline S1087/N1104L & - & WT & defect & defect & WT & WT \\
\hline R1090/1107A & - & cs & defect & - & decreased & - \\
\hline R1090/1107L & - & cs & defect & - & decreased & WT \\
\hline R1090/1107P & WT & - & defect & defect & - & - \\
\hline
\end{tabular}

Figure 1: $b r r 2-R P$ impact multiple aspects of Brr2 function during splicing. A. Structure of Brr2's N (cyan) and C (blue) cassettes(Santos et al. 2012). Amino acid positions impacted in RP are colored in red. The Prp8 Jab1 domain is shown in tan, and a modeled RNA duplex in gray. A-top right. Zoom in on positions C520 and Q904, which are located proximate to Brr2's ATPase domain. A-bottom right. Zoom in on positions N1104 and R1107 in Brr2's ratchet helix, which allows for processive dsRNA unwinding by Brr2. Figures made using pymol, PDB:4BGD (www.pymol.org). B. Spotting assay showing growth of different brr2-RP on rich media at the temperatures indicated. A subset of brr2-RP exhibit cold (R1107A, R1107L) or temperature (C520R) sensitive growth. C. Table summarizing known effects of brr2-RP. Data are included from this work, and the following references: (Zhao et al. 2009; Zhang et al. 2009; Santos et al. 2012; Mozaffari-Jovin et al. 2013; Ledoux and Guthrie 2016). 
yeast growth. We spotted serial dilutions of logphase yeast cultures onto rich media and allowed $B R R 2$ and $b r r 2-R P$ strains to grow at $37^{\circ} \mathrm{C}, 30^{\circ} \mathrm{C}, 25^{\circ} \mathrm{C}$, and $17^{\circ} \mathrm{C}$. Consistent with previous results, brr2-R1107A and brr2-R1107L exhibited cold sensitive growth at both $25^{\circ} \mathrm{C}$ and $17^{\circ} \mathrm{C}$ (Figure $\left.1 \mathrm{~B}, \mathrm{C}\right)($ Pena et al. 2009; Zhao et al. 2009; Small et al. 2006; Cordin et al. 2014; Hahn et al. 2012). Previous studies have shown that both Brr2-R1107A and Brr2-R1107L also exhibit decreased helicase activity in vitro (Figure 1C, and references indicated in figure legend). However, brr2-R1107M, which is not associated with RP, grew as wild type BRR2, clearly indicating that not all mutations at position R1107 negatively impact yeast growth (Figure 1B). Furthermore, Brr2-Q904E and Brr2-N1104L also exhibit compromised helicase activity(Zhao et al. 2009; Mozaffari-Jovin et al. 2013; Ledoux and Guthrie 2016), but neither mutation causes cold sensitivity (Figure 1C). While it is possible that helicase activity is not compromised enough by these alleles to cause cold sensitivity, it is also likely that other aspects of Brr2 function may also be negatively affected by Brr2-R1107A and Brr2-R1107L and contribute to the cold sensitive phenotype.

By contrast, brr2-C520R was temperature sensitive, exhibiting a slight but repeatable growth defect at $37^{\circ} \mathrm{C}$ (Figure 1B). To our knowledge, this is the only temperature sensitive $b r r 2-R P$ allele. We speculate that brr2C520R temperature sensitivity could result from a defect in ATP binding caused by destabilization of the Brr2 ATP-binding pocket, however more biochemical assays are required to understand how this defect arises. Together these data show that RP-associated amino acid substitutions negatively impact a wide range of Brr2 functions.

\section{Multiple endogenous pre-mRNAs exhibit intron retention in brr2-RP}

The effects of RP mutations on Brr2 growth, ATPase activity, and helicase activity that we and others have observed suggest that pre-mRNA splicing may be compromised in brr2-RP in vivo. To test this we measured the relative
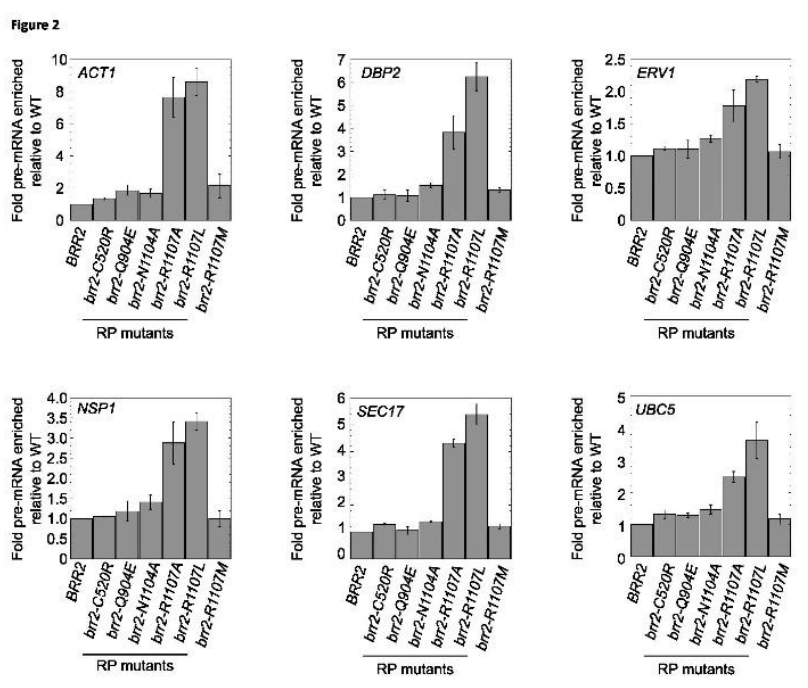

Figure 2: Multiple cellular transcripts exhibit intron retention in vivo in brr2-RP. RT-qPCR showing level of pre-mRNA accumulation in brr2-RP. brr2-R1107A and brr2-R1107L consistently exhibit elevated levels of unspliced pre-mRNA relative to wild type $B R R 2$. Experiments performed with total RNA isolated from log phase cultures grown in rich media at $30^{\circ} \mathrm{C}$.

abundancies of pre-mRNA and spliced mRNA of six transcripts (ACT1, DBP2, ERV1, NSP1, SEC17, and UBC5) by RT-qPCR. Both ACT1 and NSP1 encode pre-mRNA transcripts with introns that can be considered typical for $S$. cerevisiae in both size (308 and $118 \mathrm{nt}$, respectively) and splice-site sequence (Spingola et al. 1999). DBP2 encodes a pre-mRNA transcript with an unusually long intron (over $1000 \mathrm{nt}$ ). UBC5 and SEC17 both encode pre-mRNA transcripts with atypical aAG $3^{\prime}$ splice sites. ERV1 transcripts have a nonconsensus UAuUAAC branch site(Engel et al. 2014). In addition, while all of these genes are constitutively expressed, their relative levels of expression vary significantly(Thompson and Cubillos 2017).

We performed RT-qPCR on cDNA made from total RNA isolated from log phase wild type $B R R 2$, the five brr2-RP strains, and also the control brr2-R1107M strain grown at $30^{\circ} \mathrm{C}$ (Figure 2). Regardless of transcript identity, only brr2-R1107A and brr2-R1107L exhibit significant intron retention consistent with a defect in premRNA splicing (Figure 2). Both alleles are cold sensitive alleles (Figure 1B). However, though brr2-R1107A is slightly more cold sensitive than brr2-R1107L, in all cases the brr2-R1107L intron 
retention defect is stronger (Figure 1,2). No other brr2 alleles showed any appreciable intron retention. That there is no intron retention defect in brr2-R1107M suggests that, as with cold sensitivity, not all substitutions of amino acid R1107 are detrimental. Furthermore, though helicase activity is compromised in brr2Q904E, this strain does not exhibit cold sensitivity or intron retention (Figure 1B, C and Figure 2), suggesting that defects in helicase activity per se are not the direct or sole cause of intron retention in brr2-R1107A and R1107L, though it is also possible that the Q904E mutation simply does not have enough of an effect on helicase activity to impact intron retention. More experiments are needed to ascertain what, precisely, causes intron retention in this strain.

\section{Brr2 is a splicing fidelity factor}

Given that multiple spliceosomal DExD/H box proteins use kinetic proofreading mechanisms to ensure that splicing proceeds with high fidelity(Semlow and Staley 2012), and that RPassociated variants of human Brr2 have been linked to cryptic splicing(Cvačková et al. 2013), we were curious as to whether brr2-RP impacted splicing fidelity. We decided to use Act-Cup splicing reporters to assess whether or not brr2$R P$ affect splicing fidelity. The Act-Cup system is an established assay that reports on both splicing fidelity and efficiency(Lesser and Guthrie 1993). In this system, the well-characterized ACT1 intron is fused to the CUP1 coding sequence (Figure $3 \mathrm{~B}$ ). The Cup1 protein chelates copper in a concentration-dependent manner, allowing yeast to survive in what would otherwise be lethal copper-containing media. By fusing the ACT1 intron upstream of CUP1, this reporter directly links splicing efficiency to Cup1 expression level, which is assessed by comparing growth of brr2-RP and $B R R 2$ yeast strains transformed with the Act-Cup reporter in copper-containing media (Figure 3A). Splicing fidelity can be gauged by mutating the $5^{\prime}$ splice site, branch site, or $3^{\prime}$ splice site of the Act-Cup reporter and comparing the growth of brr2-RP to $B R R 2$ strains (Figure $3 C$ ). Increased growth indicates lower fidelity splicing, and decreased growth indicates higher fidelity and/or lower efficiency splicing.

We knocked out the genomic copy of CUP1 in our BRR2 and brr2-RP strains and transformed them with the wild type Act-Cup reporter as well as a variety of Act-Cup reporters bearing mutations in their $5^{\prime}$ splice site, branch site, and 3 ' splice site. Serial dilutions of log phase cultures of these strains were spotted onto plates supplemented with between 0 and $1.5 \mathrm{mM}$ $\mathrm{CuSO}_{4}$ and their growth at $25^{\circ} \mathrm{C}, 30^{\circ} \mathrm{C}$, and $37^{\circ} \mathrm{C}$ assessed. We determined the maximal concentration of $\mathrm{CuSO}_{4}$ that supported growth for each brr2-RP carrying each reporter [ $\mathrm{Cu}_{\max }$, brr2-RP]. We compared this value for the maximum concentration of growth tolerated by $B R R 2$ bearing the same reporter [ $\left.\mathrm{Cu}_{\max }, B R R 2\right]$, and used them to calculate a splicing index $\log _{2}\left(\left[\mathrm{Cu}_{\max }, b r r 2-R P\right] /\left(\left[\mathrm{Cu}_{\max }, b r r 2-R P\right]+\left[\mathrm{Cu}_{\max }\right.\right.\right.$, $B R R 2])$. We summarize these data as a heat map in Figure 3D. Blue coloring indicates that the indicated brr2-RP Act-Cup reporter combination grew less well than the same reporter in $B R R 2$, yellow coloring indicates better growth (Figure 3D). We included brr2-1 as a non-RP control known to show defects in dsRNA unwinding(Raghunathan and Guthrie 1998; Maeder et al. 2009).

As expected, when transformed with the wild type Act-Cup reporter, alleles that had previously shown a temperature or cold sensitive growth phenotype also exhibited decreased growth on copper, consistent with inefficient splicing. Temperature sensitive brr2C520R exhibited reduced growth at $30^{\circ} \mathrm{C}$ and $37^{\circ} \mathrm{C}$, while cold sensitive brr2-R1107A, brr2R1107L, and brr2-1 showed reduced growth at $25^{\circ} \mathrm{C}, 30^{\circ} \mathrm{C}$, and $37^{\circ} \mathrm{C}$ (Figure $3 \mathrm{D}$ column 1 ). As compared to observing brr2-RP growth without a reporter and on rich media, the expanded detrimental temperature ranges observed in this assay demonstrate its sensitivity.

The mutated reporter data are more complex. Reporter effects vary between brr2-RP strains in both the direction and magnitude of their effect 
bioRxiv preprint doi: https://doi.org/10.1101/354514; this version posted June 22, 2018. The copyright holder for this preprint (which was not certified by peer review) is the author/funder, who has granted bioRxiv a license to display the preprint in perpetuity. It is made available under aCC-BY-NC-ND 4.0 International license.

Mayerle and Guthrie, June 22, 2018 - preprint copy

Figure 3

A

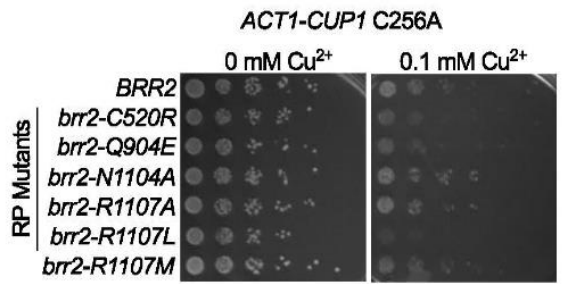

B

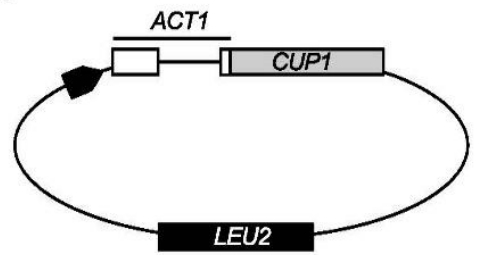

3'SS
CUP1

D

峦|

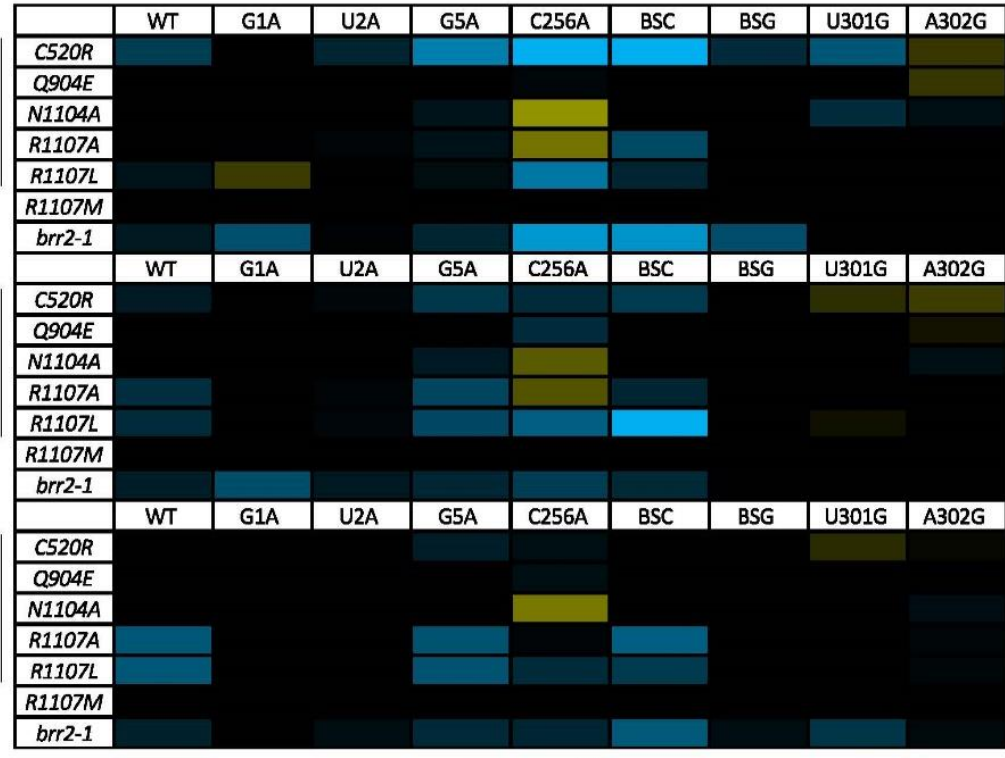

$-2.0$

Hyperaccurate $\log 2$ Error Prone

E

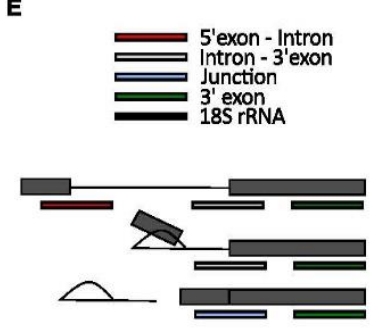

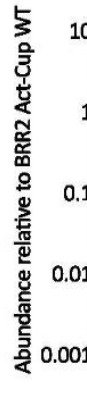

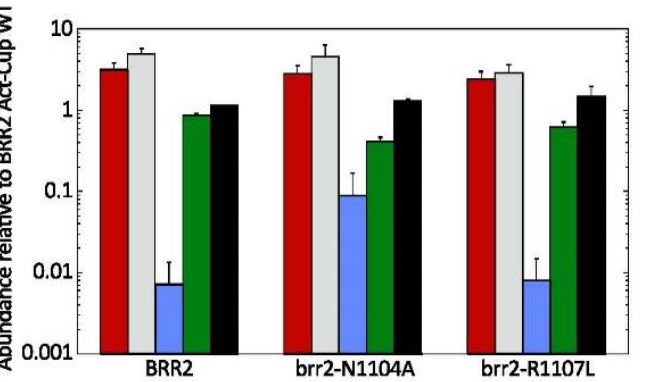

Figure 3. Brr2 is a splicing fidelity factor. A. Example of Act-Cup brr2-RP reporter assay. Indicated strains were spotted on minimal media containing $\mathrm{CuSO}_{4}$ and monitored for growth at $30^{\circ} \mathrm{C}$. The left panel shows growth of brr2-RP yeast carrying a C256A Act-Cup reporter in the presence of $0 \mathrm{mM} \mathrm{CuSO}_{4}$, the right at 0.1 $\mathrm{mM} \mathrm{CuSO}$. brr2-N1104A exhibits improved growth as compared to $B R R 2$, while brr2-R1107A and brr2-R1107L growth is impaired. B. Schematic of ActCup reporter. The $5^{\prime}$ exon and intron of ACT1 have been fused to the coding sequence of CUP1. Expression is driven by the GPD promoter. LEU2 is included in the reporter plasmid as a selectable marker. C. Schematic of Act1-Cup1 pre-mRNA. Splice-site mutations made to test how $b r r 2-R P$ impact splicing fidelity are in red. D. Heat map showing results of Act-Cup assays. Values shown were calculated by $\log _{2}\left(\left[\mathrm{Cu}_{\max } \quad\right.\right.$ Prp8-RP]/[C $\mathrm{Cu}_{\max } \quad$ Prp8-WT $\left.]\right)$ (Mayerle and Guthrie 2016). Blue indicates reduced growth in coppercontaining media (hyperaccuracy) of brr2$R P$ relative to wild type with the same reporter, while yellow indicates improved growth (error-prone splicing). E. Results of a primer extension assay monitoring the abundance of different RNA species. Primers spanning the 5 'exon intron junction (red) report on pre-mRNA abundance. Primers spanning the intron 3 'exon junction (gray) report on premRNA and lariat intermediate abundance. Primers spanning the 5'exon 3'exon junction (blue) report on spliced mRNA abundance. Primers within the $3^{\prime}$ exon (green) report on pre-mRNA, lariat intermediate, and mRNA levels. 18S rRNA was included as a control. The increased growth of brr2-N1104A carrying the C256A reporter is due to increased levels of Act-Cup mRNA. Data are the average of 3 biological measurements, error bars are SEM. Primer sequences provided in (MacRae et al. 2018).

splicing, in the presence of at least one Act-Cup reporter (Figure 3D). This pattern is unique to brr2-RP, as brr2-1 showed only reduced growth and the growth of $b r r 2-R 1107 M$ was identical to that of $B R R 2$ for all reporters tested, consistent with its behavior in previous assays (Figure 3D). on growth (Figure 3D). Every single brr2-RP strain exhibited better growth (yellow coloring), indicative of error-prone splicing, in the presence of at least one Act-Cup reporter, though in some cases the effect was very slight. Furthermore, every single brr2-RP strain exhibited worse growth (blue coloring), indicative of inefficient or hyperaccurate 


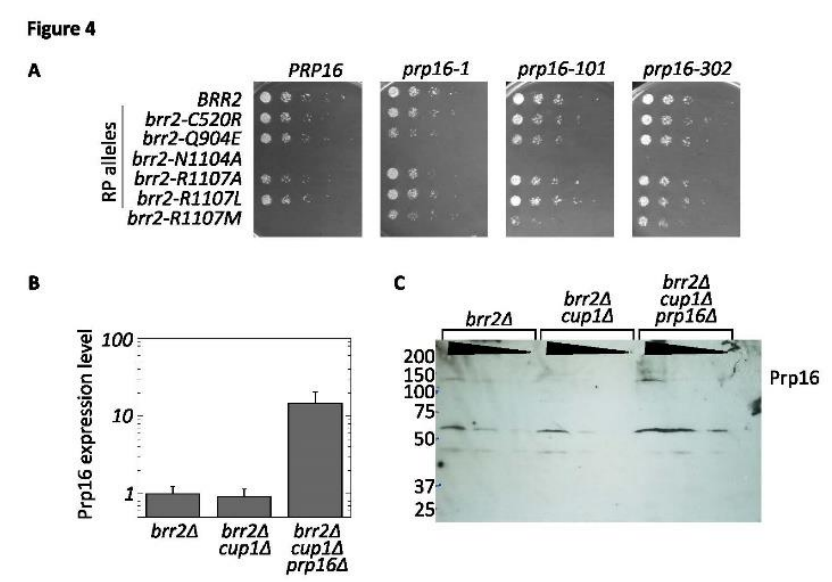

The most interesting patterns occur in the presence of the C256A branch site mutant ActCup reporter. This reporter negatively affects the growth of brr2-C520R, brr2-R1107L, brr2$R 1107 M$, and brr2-1 at all temperatures consistent with inefficient or hyperaccurate splicing. At the same time, brr2-N1104A and brr2-R1107A growth is improved, indicative of error-prone splicing (Figure 3D, column 5). brr2Q904E and brr2-R1107M grew as wild type BRR2. qRT-PCR analysis of the relative abundances of Act-Cup reporter pre-mRNA, lariat-intermediate, and $\mathrm{mRNA}$ are consistent with these growth data (Figure 3E). These data show that, at least in the case of brr2-RP, splicing fidelity is not directly linked to helicase activity or ATPase activity (Figure 1C, Figure 3D). Together our Act-Cup reporter data clearly implicate Brr2 in splicing fidelity, however the mechanism underlying its effects remains unclear.

\section{Prp16 ATPase activity and expression levels impact Brr2 function}

While Brr2 has been shown to interact physically and genetically with Prp16(Fromont-Racine et al. 1997; Dix et al. 1998; van Nues and Beggs 2001; Liu et al. 2006; Hahn et al. 2012; Cordin et al. 2014; Absmeier et al. 2015; Galej et al. 2016), how these two proteins influence each other is unknown. Because both Brr2 and Prp16 are involved in proofreading the pre-mRNA branch site (Figure 3) (Burgess and Guthrie 1993a), and Prp16 ATPase activity has been directly linked to its proofreading activity(Burgess and Guthrie 1993b), we decided to determine if Prp16-
Figure 4. $b r r 2-R P$ are sensitive to Prp16 abundance and ATPase activity. A. Growth assay. brr2 prp16 double mutant yeast containing the indicated alleles were spotted onto rich media and allowed to grow at $30^{\circ} \mathrm{C}$. In these yeast, the genomic copies of $B R R 2$ and $P R P 16$ have been knocked out and Brr2 and Prp16 are expressed from plasmids. In this strain background brr2-N1104A is lethal in combination with $P R P 16$ and all prp16 alleles. brr2-R1107M is similarly lethal when combined with PRP16 in this strain background, however, lethality can be rescued by ATPase deficient prp16 alleles. B. RT-qPCR comparing PRP16 transcript abundance (Primers: Prp16F 5' AGGGCAAGAGACATTCGAGA 3', Prp16R 5' TGCTTGATGAGCAAATCCTG $\left.3^{\prime}\right)$. The strain used in panel A has increased PRP16 mRNA abundance, likely due to its expression from a plasmid, not the genomic copy of PRP16. C. Western blot probed with polyclonal Prp16 antibody (Burgess and Guthrie 1993a) showing elevated Prp16 protein expression in the strain used in panel A. A nonspecific band at $60 \mathrm{KDa}$ serves as a loading control.

ATPase deficient alleles genetically interact with brr2-RP. We created double mutant strains in which the genomic copies of both BRR2 and PRP16 are deleted and Brr2 and Prp16 are expressed from plasmids. We then shuffled in plasmids containing $B R R 2$ or brr2-RP and $P R P 16$ or a prp16 ATPase deficient allele. Finally we plated these strains on rich media and assessed their growth at $30^{\circ} \mathrm{C}$.

Unexpectedly, we observed synthetic lethality with brr2-N1104A and brr2-R1107M in combination with wild type PRP16 when both proteins were expressed from plasmids (Figure 4A). Both Brr2 and Prp16 are expressed from low-copy plasmids in this strain, and both are under the control of their native promoters. However neither BRR2 nor PRP16 are expressed in their full genetic and chromosomal context. We therefore speculated that the synthetic lethality observed may be due to fact that Prp16 is overexpressed in this strain background. We assessed Prp16 expression at the mRNA level by RT-qPCR, and at the protein level by western blot with a polyclonal anti-Prp16 antibody. At both the mRNA and protein levels, Prp16 is overexpressed in the brr2 prp16 double mutant strain as compared to strains where only Brr2 is expressed from a plasmid (Figure 4B,C). More intriguingly, brr2-R1107M PRP16 synthetic lethality is rescued by ATPase deficient alleles of prp16, though these stains are clearly still sick (Figure 4A). All other combinations of brr2-RP and prp16 alleles showed no genetic 
interactions. The interactions between brr2N1104A and brr2-R1107M and PRP16 and its ATPase deficient alleles do not correlate with any other phenotype tested, suggesting that erroneous interactions with Prp16 may be another way that brr2-RP negatively affects splicing. Because brr2-R1107M PRP16 lethality is rescued by prp16 ATPase alleles, these data suggest a functional link between Brr2 and Prp16 ATPase activity. However, more work is required to fully understand the mechanism underlying these genetic observations.

\section{Conclusion}

Together these data implicate Brr2 in splicing fidelity, and highlight a previously unknown connection between Brr2 and Prp16 ATPase activity. They further implicate error-prone splicing in SNRNP200-related RP pathogenesis. More study is needed to understand how Brr2 impacts splicing fidelity, how the activities of Brr2 and Prp16 are coordinated, and what the functional consequences of disrupting these Brr2 functions are in Retinitis Pigmentosa.

\section{Materials and Methods}

Strains and Plasmids. Strains and plasmids were constructed using standard cloning techniques(Guthrie and Fink 2004). Act-Cup reporter plasmids were described in (Lesser and Guthrie 1993). All plasmids referenced in this manuscript, including the Act-Cup reporter plasmids, are available from Addgene (www.addgene.org). Strain requests should be directed to Hiten Madhani.

\begin{tabular}{l|l} 
STRAIN & \multicolumn{1}{l}{ GENOTYPE } \\
\hline YMEG022 & brr2::KAN his leu lys ura yCP50-BRR2 \\
YMEG010 & $\begin{array}{l}\text { brr2::KAN cup1::ura3-52 his leu lys ura } \\
\text { ade yCP50-BRR2 } \\
\text { brr2::KAN prp16::LYS cup1::ura3-52 } \\
\text { ade met leu his yCP50-BRR2 pRS316- } \\
\text { PRP16 }\end{array}$
\end{tabular}

Yeast growth assays. For competitive growth assays, saturated overnight yeast cultures were diluted to $O D_{600}$ of 0.1 in rich media and allowed to grow until they reached mid-log phase. Then the cultures were serially diluted and spotted onto rich media. Plates were allowed to grow at the indicated temperatures and then photographed.

$R T$-qPCR. In all cases, total RNA was isolated from mid-log yeast cultures and prepped using the hot phenol method, treated with DNase, and then phenol-chloroform extracted again. cDNA was created using dN9 primers and Superscript III Reverse Transcriptase using the manufacturers protocols. Methods and primers for RT-qPCR of Act-Cup reporter cDNA are described in (MacRae et al. 2018), methods and primers for RT-qPCR of endogenous introncontaining transcripts are described in (Mayerle and Guthrie 2016). Prp16 primers are noted in the legend of Figure 4.

ACT-CUP reporter assays. The Act-Cup reporter system has been described previously (Lesser and Guthrie 1993). The experiments described in this manuscript were performed using the same protocol as described in (Mayerle and Guthrie 2016).

Western blot. Western blot analysis of Prp16 protein abundance was performed using the antibodies and method described in (Burgess and Guthrie 1993a).

\section{Acknowledgements}

We thank everyone who has provided input into the interpretation of these data, particularly John Abelson, Jean Beggs, Corina Maeder, Magda Konarska, Charles Query, Jon Staley, Joan Steitz, Hiten Madhani, Jordan Burke, Sara Espinosa, and the UCSC RNA Club. 


\section{References}

Absmeier E, Becke C, Wollenhaupt J, Santos KF, Wahl MC. 2017. Interplay of cis- and transregulatory mechanisms in the spliceosomal RNA helicase Brr2. Cell Cycle Georget Tex 16: 100112.

Absmeier E, Rosenberger L, Apelt L, Becke C, Santos KF, Stelzl U, Wahl MC. 2015. A noncanonical PWI domain in the N-terminal helicase-associated region of the spliceosomal Brr2 protein. Acta Crystallogr D Biol Crystallogr 71: 762-771.

Burgess SM, Guthrie C. 1993a. A mechanism to enhance mRNA splicing fidelity: the RNAdependent ATPase Prp16 governs usage of a discard pathway for aberrant lariat intermediates. Cell 73: 1377-1391.

Burgess SM, Guthrie C. 1993b. Beat the clock: paradigms for NTPases in the maintenance of biological fidelity. Trends Biochem Sci 18: 381384.

Chen H-C, Tseng C-K, Tsai R-T, Chung C-S, Cheng S-C. 2013. Link of NTR-mediated spliceosome disassembly with DEAH-box ATPases Prp2, Prp16, and Prp22. Mol Cell Biol 33: 514-525.

Cordin O, Hahn D, Alexander R, Gautam A, Saveanu C, Barrass JD, Beggs JD. 2014. Brr2p carboxy-terminal Sec63 domain modulates Prp16 splicing RNA helicase. Nucleic Acids Res 42: 13897-13910.

Cvačková Z, Matějů D, Staněk D. 2013. Retinitis Pigmentosa Mutations of SNRNP200 Enhance Cryptic Splice-Site Recognition. Hum Mutat n/an/a.

Daiger SP, Bowne SJ, Sullivan LS. 2014. Genes and Mutations Causing Autosomal Dominant Retinitis Pigmentosa. Cold Spring Harb Perspect Med.

Dix I, Russell CS, O'Keefe RT, Newman AJ, Beggs JD. 1998. Protein-RNA interactions in the U5
snRNP of Saccharomyces cerevisiae. RNA 4: 1675-1686.

Engel SR, Dietrich FS, Fisk DG, Binkley G, Balakrishnan R, Costanzo MC, Dwight SS, Hitz BC, Karra K, Nash RS, et al. 2014. The reference genome sequence of Saccharomyces cerevisiae: then and now. G3 Bethesda Md 4: 389-398.

Fox-Walsh KL, Hertel KJ. 2009. Splice-site pairing is an intrinsically high fidelity process. Proc Natl Acad Sci U S A 106: 1766-1771.

Fromont-Racine M, Rain JC, Legrain P. 1997. Toward a functional analysis of the yeast genome through exhaustive two-hybrid screens. Nat Genet 16: 277-282.

Galej WP, Wilkinson ME, Fica SM, Oubridge C, Newman AJ, Nagai K. 2016. Cryo-EM structure of the spliceosome immediately after branching. Nature.

Guthrie C, Fink GR. 2004. Guide to Yeast Genetics and Molecular and Cell Biology. Gulf Professional Publishing.

Hahn D, Kudla G, Tollervey D, Beggs JD. 2012. Brr2p-mediated conformational rearrangements in the spliceosome during activation and substrate repositioning. Genes Dev 26: 2408-2421.

Lauber J, Fabrizio P, Teigelkamp S, Lane WS, Hartmann E, Luhrmann R. 1996. The HeLa 200 kDa U5 snRNP-specific protein and its homologue in Saccharomyces cerevisiae are members of the DEXH-box protein family of putative RNA helicases. EMBO J 15: 4001-4015.

Ledoux S, Guthrie C. 2016. Retinitis Pigmentosa Mutations in Bad Response to Refrigeration 2 (Brr2) Impair ATPase and Helicase Activity. J Biol Chem 291: 11954-11965.

Lesser CF, Guthrie C. 1993. Mutational analysis of pre-mRNA splicing in Saccharomyces cerevisiae using a sensitive new reporter gene, CUP1. Genetics 133: 851-863. 
bioRxiv preprint doi: https://doi.org/10.1101/354514; this version posted June 22, 2018. The copyright holder for this preprint (which was not

Mayerle and Guthrie, June 22, 2018 - preprint copy

Liu S, Rauhut R, Vornlocher H-P, Lührmann R. 2006. The network of protein-protein interactions within the human U4/U6.U5 trisnRNP. RNA N Y N 12: 1418-1430.

MacRae AJ, Mayerle M, Hrabeta-Robinson E, Chalkley RJ, Guthrie C, Burlingame AL, Jurica M. 2018. Prp8 positioning of U5 snRNA is linked to 5' splice site recognition. RNA NYN.

Maeder C, Kutach AK, Guthrie C. 2009. ATPdependent unwinding of U4/U6 snRNAs by the Brr2 helicase requires the C-terminus of Prp8. Nat Struct Mol Biol 16: 42-48.

Mayerle M, Guthrie C. 2016. Prp8 retinitis pigmentosa mutants cause defects in the transition between the catalytic steps of splicing. RNA N YN.

Mozaffari-Jovin S, Wandersleben T, Santos KF, Will CL, Lührmann R, Wahl MC. 2013. Inhibition of RNA helicase Brr2 by the C-terminal tail of the spliceosomal protein Prp8. Science 341: 8084.

Nguyen THD, Li J, Galej WP, Oshikane H, Newman AJ, Nagai K. 2013. Structural Basis of Brr2-Prp8 Interactions and Implications for U5 snRNP Biogenesis and the Spliceosome Active Site. Struct England1993 21: 910-919.

Noble SM, Guthrie C. 1996. Identification of novel genes required for yeast pre-mRNA splicing by means of cold-sensitive mutations. Genetics 143: 67-80.

Pena $V$, Jovin SM, Fabrizio $P$, Orlowski J, Bujnicki JM, Lührmann R, Wahl MC. 2009. Common design principles in the spliceosomal RNA helicase Brr2 and in the Hel308 DNA helicase.

Mol Cell 35: 454-466.

Raghunathan PL, Guthrie C. 1998. RNA unwinding in U4/U6 snRNPs requires ATP hydrolysis and the DEIH-box splicing factor Brr2. Curr Biol CB 8: 847-855.
Růžičková Š, Staněk D. 2017. Mutations in spliceosomal proteins and retina degeneration. RNA Biol 14: 544-552.

Santos KF, Jovin SM, Weber G, Pena V, Lührmann R, Wahl MC. 2012. Structural basis for functional cooperation between tandem helicase cassettes in Brr2-mediated remodeling of the spliceosome. Proc Natl Acad Sci U S A 109: 17418-17423.

Semlow DR, Staley JP. 2012. Staying on message: ensuring fidelity in pre-mRNA splicing. Trends Biochem Sci 37: 263-273.

Small EC, Leggett SR, Winans AA, Staley JP. 2006. The EF-G-like GTPase Snu114p regulates spliceosome dynamics mediated by Brr2p, a DExD/H box ATPase. Mol Cell 23: 389-399.

Spingola M, Grate L, Haussler D, Ares M. 1999. Genome-wide bioinformatic and molecular analysis of introns in Saccharomyces cerevisiae. RNA N Y N 5: 221-234.

Thompson DA, Cubillos FA. 2017. Natural gene expression variation studies in yeast. Yeast Chichester Eng/ 34: 3-17.

van Nues RW, Beggs JD. 2001. Functional contacts with a range of splicing proteins suggest a central role for Brr2 $p$ in the dynamic control of the order of events in spliceosomes of Saccharomyces cerevisiae. Genetics 157: 1451-1467.

Will CL, Lührmann R. 2011. Spliceosome structure and function. Cold Spring Harb Perspect Biol 3.

Zhao C, Bellur DL, Lu S, Zhao F, Grassi MA, Bowne SJ, Sullivan LS, Daiger SP, Chen LJ, Pang $\mathrm{CP}$, et al. 2009. Autosomal-dominant retinitis pigmentosa caused by a mutation in SNRNP200, a gene required for unwinding of U4/U6 snRNAs. Am J Hum Genet 85: 617-627. 